\title{
DIOPHANTINE APPROXIMATION BY PRIMES
}

\author{
KAISA MATOMÄKI \\ Department of Mathematics, University of Turku, 20014 Turku, Finland \\ e-mail:ksmato@utu.fi
}

(Received 6 November 2008; accepted 1 June 2009)

\begin{abstract}
We show that whenever $\delta>0$ and constants $\lambda_{i}$ satisfy some necessary conditions, there are infinitely many prime triples $p_{1}, p_{2}, p_{3}$ satisfying the inequality $\left|\lambda_{0}+\lambda_{1} p_{1}+\lambda_{2} p_{2}+\lambda_{3} p_{3}\right|<\left(\max p_{j}\right)^{-2 / 9+\delta}$. The proof uses Davenport-Heilbronn adaption of the circle method together with a vector sieve method.
\end{abstract}

2000 Mathematics Subject Classification. 11D75, 11N36, 11P32.

1. Introduction. We prove the following theorem. It can be seen as a quantitative Diophantine approximation version of the ternary Goldbach conjecture.

THEOREM 1. Suppose that $\lambda_{1}, \lambda_{2}$ and $\lambda_{3}$ are non-zero real numbers, not all of the same sign, that $\lambda_{0}$ is real, and that $\lambda_{1} / \lambda_{2}$ is irrational. Let $\xi=2 / 9$ and $\delta>0$. Then there are infinitely many ordered triples of primes $p_{1}, p_{2}, p_{3}$ for which

$$
\left|\lambda_{0}+\lambda_{1} p_{1}+\lambda_{2} p_{2}+\lambda_{3} p_{3}\right|<\left(\max p_{j}\right)^{-\xi+\delta} .
$$

Results of this type were first obtained by Schwarz [14] and Baker [1]. Vaughan [15] was the first one who managed to prove (1) with a polynomial saving (with $\xi=1 / 10$ ). The exponent was subsequently improved by Baker and Harman [2] to $\xi=1 / 6$ and by Harman [7] to $\xi=1 / 5$. Baker and Harman [2] also showed that under the generalised Riemann hypothesis (1) holds with $\xi=1 / 4$.

Our method goes back to Vaughan [15] but we take advantage of an averaging following [7]. The basic method builds on the Davenport-Heilbronn adaptation of the circle method (see [16, Chapter 11]). Our improvement comes from using a sieve method developed by Harman $[\mathbf{6}, \mathbf{8}, \mathbf{9}$ ] combined with a vector sieve. For an earlier work combining these see [3]. To be able to get our improved result, we also prove and use slightly improved versions of estimates for averaged exponential sums in [7].

Let $q^{\prime}$ be a large enough (in terms of $\lambda_{i}$ ) denominator of a convergent to $\lambda_{1} / \lambda_{2}$. We write $X=q^{1 /(3 \xi)}$ and $\epsilon=X^{-\xi+\delta}$. Then $q^{\prime}=\epsilon^{-3} X^{3 \delta}=X^{2 / 3}$.

Let further

$$
K_{\epsilon}(x)=\left(\frac{\sin (\pi \epsilon x)}{\pi x}\right)^{2} .
$$

Then $K_{\epsilon}(x) \ll \min \left\{\epsilon^{2}, x^{-2}\right\}$. The basis of the Davenport-Heilbronn adaption of the circle method is the identity

$$
\max \{0, \epsilon-|x|\}=\int_{-\infty}^{\infty} K_{\epsilon}(y) e(x y) d y .
$$


To set up a vector sieve, we need lower and upper bounds $\rho^{-}(n)$ and $\rho^{+}(n)$ for the characteristic function $\rho(n)$ of primes. Assuming $\rho^{-}(n) \leq \rho(n) \leq \rho^{+}(n)$ we have a simple inequality

$$
\rho(m) \rho(n) \geq \rho^{+}(m) \rho^{-}(n)+\rho^{-}(m) \rho^{+}(n)-\rho^{+}(m) \rho^{+}(n)
$$

given in [4].

We will choose the coefficients $\rho^{ \pm}(n)$ so that they are divisor-bounded, as will be all later appearing coefficients $a_{n}, b_{n}, \ldots$. This means, for example, that $a_{n} \ll \tau(n)^{C}$ for some constant $C$.

We write for $i=1,2$

$$
S_{i}^{+}(x)=\sum_{n \asymp X} \rho^{+}(n) e\left(n \lambda_{i} x\right), \quad S_{i}^{-}(x)=\sum_{n \asymp X} \rho^{-}(n) e\left(n \lambda_{i} x\right)
$$

and

$$
S_{3}(x)=\sum_{n \asymp X} \rho(n) e\left(n \lambda_{3} x\right) .
$$

Here $n \asymp X$ means that $n \in[d X, D X]$, where $d$ and $D$ are such that the equation $\lambda_{1} x_{1}+\lambda_{2} x_{2}+\lambda_{3} x_{3}=0$ has solutions with $x_{i} \in(d, D)$. Such pairs $(d, D)$ exist because $\lambda_{1}, \lambda_{2}$ and $\lambda_{3}$ are not all of the same sign.

We define further

$$
F(x)=S_{1}^{+}(x) S_{2}^{-}(x) S_{3}(x)+S_{1}^{-}(x) S_{2}^{+}(x) S_{3}(x)-S_{1}^{+}(x) S_{2}^{+}(x) S_{3}(x) .
$$

Then the basis of our method is the following implication of (2) and (3).

LEMMA 2. The number of solutions to (1) with $p_{j} \asymp X$ is

$$
\geq \epsilon^{-1} \int_{-\infty}^{\infty} K_{\epsilon}(x) F(x) e\left(x \lambda_{0}\right) d x .
$$

2. Further description of the method. Throughout the paper, we use the letter $\eta$ for a small (in terms of $\delta$ ) positive constant that might not be the same at each occurrence. However, it would be possible to give all the constants $\eta$ exact values that belong to $\{k \delta / 1000 \mid 1 \leq k \leq 100\}$, say.

We write $\tau=X^{-1+\eta} \epsilon^{-1}=X^{\xi-\delta-1+\eta}$ and $P=\epsilon^{-2} X^{\eta}$ and divide the integral in (4) into three regions $|x| \leq \tau, \tau<|x| \leq P$ and $|x|>P$. The last integral is almost trivially $\ll \epsilon^{2} X^{2-\eta}$ as in Section 7 of [15].

In the major arc we need Gallagher's lemma [5, Lemma 1].

LEMMA 3. Let

$$
S(t)=\sum_{v} a_{v} e(v t)
$$

be an absolutely convergent exponential sum and let $\delta>0$. Then

$$
\int_{-\delta}^{\delta}|S(t)|^{2} d t \ll \int_{-\infty}^{\infty}\left|\delta \sum_{v=x}^{x+1 /(2 \delta)} a_{v}\right|^{2} d x .
$$


Now we get a non-trivial lower bound for the integral over the major arc.

Lemma 4. Assume that there are positive real numbers $u^{-}$and $u^{+}$with $2 u^{-}>u^{+}$ such that for any $\vartheta \in\left[\frac{1}{6 \tau X}, \frac{6}{\tau X}\right]$ and $A \geq 0$, we have

$$
\int_{d X}^{D X}\left(\sum_{y \leq m<y+y \vartheta}\left(\rho^{ \pm}(m)-\frac{u^{ \pm}}{\log m}\right)\right)^{2} d y \ll \frac{X}{\tau^{2}}(\log X)^{-A}
$$

Then

$$
\int_{-\tau}^{\tau} K_{\epsilon}(x) F(x) e\left(x \lambda_{0}\right) d x \gg \epsilon^{2} \frac{X^{2}}{(\log X)^{3}} .
$$

Proof. Define

$$
G(x)=\left(2 u^{+} u^{-}-u^{+2}\right) \prod_{j=1}^{3} I\left(\lambda_{j} x\right)
$$

where

$$
I(x)=\int_{d X}^{D X} \frac{e(x y)}{\log y} d y
$$

Then by rearranging and using the Cauchy-Schwarz inequality

$$
\begin{aligned}
& \int_{-\tau}^{\tau}|F(x)-G(x)| d x \\
& \ll X^{3 / 2}(\log X)^{C}\left(\int_{-\tau}^{\tau}\left|S_{1}^{+}(x)-u^{+} I\left(\lambda_{1} x\right)\right|^{2}+\left|S_{2}^{+}(x)-u^{+} I\left(\lambda_{2} x\right)\right|^{2}\right. \\
& \left.\quad+\left|S_{1}^{-}(x)-u^{-} I\left(\lambda_{1} x\right)\right|^{2}+\left|S_{2}^{-}(x)-u^{-} I\left(\lambda_{2} x\right)\right|^{2}+\left|S_{3}(x)-I\left(\lambda_{3} x\right)\right|^{2} d x\right)^{1 / 2} .
\end{aligned}
$$

Then (5) and a corresponding bound for $\rho(m)$ (which follows from Huxley's prime number theorem [12, Theorem 10.5 and its proof]) imply by using Lemma 3 and arguing as in [13, Lemma 6 and 7] that

$$
\int_{-\tau}^{\tau}|F(x)-G(x)| d x \ll X^{2}(\log X)^{-A}
$$

Then the proof of the claim follows by showing that

$$
\int_{-\tau}^{\tau} K_{\epsilon}(x) G(x) e\left(x \lambda_{0}\right) d x \gg \frac{\epsilon^{2} X^{2}}{(\log X)^{3}},
$$

which follows as [15, Lemma 10]. 
We will use a sieve method to choose $\rho^{+}(n)$ and $\rho^{-}(n)$ such that they can be written as sums of coefficients $a_{n}$ that are either of the form

$$
a_{n}=\sum_{\substack{m k=n \\ m \sim M}} b_{m}
$$

with $M \ll X^{7 / 9}$ (type I sums) or such that, for any $Q \in\left[X^{1 / 3}, X^{4 / 9}\right]$, there exists $M \in$ $\left[Q, Q X^{1 / 9}\right]$ such that

$$
a_{n}=\sum_{\substack{l m=n \\ m \sim M}} b_{m} c_{l}
$$

(type II sums). Here $m \sim M$ means $M \leq m<2 M$. The condition for type II sums is clearly equivalent to the assumption that $a_{n}$ can be written in form (6) with $M=M_{1} \in$ $\left[X^{4 / 9}, X^{5 / 9}\right]$ and with $M=M_{2} \in\left[X^{1 / 3}, X^{4 / 9}\right]$ satisfying $M_{1} / M_{2} \leq X^{1 / 9}$.

REMARK 5. For a general $\xi$ the bound for type I sums would be $M \leq X^{1-\eta} \epsilon$ and the width of the type II information would be $X^{1-\eta} \epsilon^{4}$. The upper bound for $Q$ would be $X^{\eta} \epsilon^{-2}$ and the interval must cover those values of $Q$ for which Lemma 14 below is used.

We write for $i=1,2$,

$$
S_{i}(x)=\sum_{n \asymp X} a_{n} e\left(n \lambda_{i} x\right)
$$

where $a_{n}$ is of one of the above types.

Thus we have to show besides (5) only that

$$
\int_{\tau}^{P}\left|S_{1}(x) S_{2}(x) S_{3}(x) K_{\epsilon}(x)\right| d x \ll \epsilon^{2} X^{2}(\log X)^{-A} .
$$

This follows from

Proposition 6. We can write $[1, P]=\mathcal{A}_{1} \cup \mathcal{A}_{2} \cup \mathcal{A}_{3}$ such that

(i) If $|x| \in \mathcal{A}_{1}$, then $\min \left\{\left|S_{1}(x)\right|,\left|S_{2}(x)\right|,\left|S_{3}(x)\right|\right\} \leq \epsilon X^{1-\eta}$;

(ii)

$$
\int_{|x| \in \mathcal{A}_{2}}\left|S_{1}(x) S_{2}(x)\right|^{2}\left|K_{\epsilon}(x)\right| d x \ll \epsilon^{3} X^{3-\eta}
$$

(iii)

$$
\int_{|x| \in \mathcal{A}_{3}}\left|S_{1}(x) S_{2}(x) S_{3}(x) K_{\epsilon}(x)\right| d x \ll \epsilon^{2} X^{2-\eta} .
$$

Furthermore, if $|x| \in[\tau, 1]$, then $\min \left\{\left|S_{1}(x)\right|,\left|S_{2}(x)\right|\right\} \leq X^{1-\eta}$. 
3. Auxiliary results. We have the following result for exponential sums.

Lemma 7. Suppose that $|x-a / q|<1 / q^{2}$ for some integers $a, q$ with $q \geq 1$ and $(a, q)=1$. Then, for any complex sequences $b_{m}, c_{l} \ll 1$, we have

$$
\begin{gathered}
\sum_{\substack{l m \sim Y \\
m \sim M}} b_{m} c_{l} e(m l x) \ll\left(Y q^{-1 / 2}+(Y q)^{1 / 2}+Y M^{-1 / 2}+(Y M)^{1 / 2}\right)(\log Y)^{2}, \\
\sum_{\substack{l m \sim Y \\
m \sim M}} b_{m} e(m l x) \ll\left(M+Y q^{-1}+q\right)(\log (2 q Y))
\end{gathered}
$$

and

$$
\sum_{p \sim Y} e(p x) \ll\left((Y q)^{1 / 2}+Y q^{-1 / 2}+Y^{4 / 5}\right)(\log Y)^{3} .
$$

Proof. See for example [12, Section 13.5].

We also need classical mean value results for Dirichlet polynomials.

LEMMA 8. We have

$$
\sum_{\chi(\bmod q)} \int_{0}^{T}\left|\sum_{n \sim N} a_{n} \chi(n) n^{i t}\right|^{2} d t \ll(N+q T)(\log N)^{3} \sum_{n \sim N}\left|a_{n}\right|^{2}
$$

and

$$
\sum_{q \leq Q} \sum_{(\bmod q)}^{*} \int_{0}^{T}\left|\sum_{n \sim N} a_{n} \chi(n) n^{i t}\right|^{2} d t \ll\left(N+Q^{2} T\right)(\log N)^{3} \sum_{n \sim N}\left|a_{n}\right|^{2},
$$

where here and later $*$ indicates that summation is over primitive characters.

Proof. These follow for example from [12, Theorem 9.12].

We write || || for the distance from the nearest integer. We need the following lemma in the course of the proof of an average result for exponential sums.

Lemma 9. Let $h \leq Q, M \geq 1$ and $\delta>0$. Let further $\mathcal{Q}$ be a set of distinct integers contained in $[Q, 2 Q)$ and for each $q \in \mathcal{Q}, a(q)$ be an integer satisfying $\operatorname{gcd}(a(q), q)=1$. Define $\mathcal{Q}_{h}=\{q \mid q h \in \mathcal{Q}\}$ and

$$
\begin{aligned}
& \mathcal{A}_{\delta, h}=\{(q, m) \in \mathcal{Q} \times[M, 2 M) \mid \operatorname{gcd}(q, m)=h, \\
& \left.\min _{\substack{\left(q_{1}, m_{1}\right) \in \mathcal{Q} \times[M, 2 M) \\
q_{1}=q \underset{\operatorname{gcd}\left(q_{1}, m_{1}\right)=h}{\Longrightarrow} m_{1} \neq m(\bmod q)}}\left\|\frac{m a(q)}{q}-\frac{m_{1} a\left(q_{1}\right)}{q_{1}}\right\|<\delta\right\} .
\end{aligned}
$$

Then

$$
\left|\mathcal{A}_{\delta, h}\right| \leq \frac{8\left|\mathcal{Q}_{h}\right|^{2}(M+Q) \delta Q}{h^{2}}
$$


Proof. Clearly, we can assume that $h=1$ since the result for $h>1$ follows from the one with $h=1$ by considering the set $\mathcal{Q}_{h} \times[M / h, 2 M / h]$.

We write $\mathcal{A}_{\delta}=\mathcal{A}_{\delta, 1}$. If $(q, m) \in \mathcal{A}_{\delta}$, then there exists $\left(q_{1}, m_{1}\right) \in \mathcal{A}_{\delta}$ with either $q_{1} \neq q$ or $m_{1} \neq \equiv m(\bmod q)$ such that

$$
\left\|\frac{m a(q)}{q}-\frac{m_{1} a\left(q_{1}\right)}{q_{1}}\right\|<\delta \Longleftrightarrow m a(q) q_{1}-m_{1} a\left(q_{1}\right) q \equiv k\left(\bmod q q_{1}\right)
$$

for some $|k|<\delta q q_{1}$. Since $\operatorname{gcd}\left(m_{1} a\left(q_{1}\right), q_{1}\right)=\operatorname{gcd}(m a(q), q)=1$, conditions on $q_{1}$ and $m_{1}$ let us assume that $k \neq 0$.

Fix $q_{1}, q \in \mathcal{Q}$. If (12) holds, then

$$
m a(q) q_{1} \equiv k(\bmod q)
$$

This is soluble only if $\operatorname{gcd}\left(q_{1}, q\right) \mid k$. In that case it has a unique solution $\left(\bmod q / \operatorname{gcd}\left(q_{1}, q\right)\right)$. Thus there are at most

$$
\frac{M \operatorname{gcd}\left(q, q_{1}\right)}{q}+1
$$

possible values $m \in[M, 2 M]$. Thus for fixed $q, q_{1} \in \mathcal{Q}$, the inequality in (12) has at most

$$
\frac{2 \delta q q_{1}}{\left(q_{1}, q\right)}\left(\frac{M\left(q_{1}, q\right)}{q}+1\right) \leq 8 \delta Q(M+Q)
$$

solutions. This proves the lemma.

LEMMA 10. Suppose that $x$ is a real number with $|x-a / q| \leq q^{-2}$ for some integers $a, q, q \geq 1$ with $(a, q)=1$. Let $A$ and $Q$ be positive integers with $A Q \ll q^{C}$ and let $\mathcal{Q}$ be a set of distinct integers $q_{1}$ with $q_{1} \sim Q$. Then, for every $\eta>0$ and $\theta<1 / 2$ the number of solutions to

$$
\left\|q_{1} n x\right\|<\theta \quad \text { with } q_{1} \in \mathcal{Q}, 1 \leq n \leq A
$$

is

$$
\ll|\mathcal{Q}| A \theta+q^{\eta}\left(Q+A Q q^{-1}+q \theta\right),
$$

where the implied constant depends only on $x, C$ and $\eta$.

Proof. The proof is as that of Lemma 4 of [7]. We get an additional term $A Q q^{\eta-1}$ since we did not assume that $A, Q \leq q$.

In the following lemma $S \subset \mathbb{R}$ may have repetitions, so we call it a multiset. We use the notation $\sum(S)=\sum_{x \in S} x$ and $S=S_{1} \cup S_{2}$ means that $S$ is a disjoint union of the multisets $S_{1}$ and $S_{2}$. If for example $S=\{1,2,2\}$, then $\sum(S)=5$ and $\{1,2\} \neq S=$ $\{1,2\} \cup \dot{\cup}\{2\}$.

LEMMA 11. Let $n>k$ and $S=\left\{\alpha_{1}, \ldots, \alpha_{n}\right\}$ with

$$
0<\alpha_{n} \leq \alpha_{n-1} \leq \cdots \leq \alpha_{k+1} \leq 1 / 9<\alpha_{k} \leq \cdots \leq \alpha_{1}<2 / 9 .
$$


We assume that the multiset $S$ satisfies the following three conditions

(i) $\sum(S)=1$;

(ii) $\alpha_{1}+\alpha_{k+1}+\alpha_{k+2}+\cdots+\alpha_{n}>2 / 9$;

(iii) $S=S_{1} \cup S_{2}$, where $\sum\left(S_{1}\right) \in[4 / 9,5 / 9]$.

Then we can write

$$
S=S_{1}^{\prime} \dot{\cup} S_{2}^{\prime}=S_{1}^{\prime \prime} \dot{\cup} S_{2}^{\prime \prime},
$$

where

$$
\sum\left(S_{1}^{\prime}\right) \in[1 / 3,4 / 9], \quad \sum\left(S_{1}^{\prime \prime}\right) \in[4 / 9,5 / 9] \quad \text { and } \quad \sum\left(S_{1}^{\prime \prime}\right)-\sum\left(S_{1}^{\prime}\right) \leq 1 / 9 .
$$

Proof. If $\sum_{i=k+1}^{n} \alpha_{i} \geq 1 / 9$, the claim is easily verified. Otherwise, by combining $\alpha_{k+1}, \ldots, \alpha_{n}$, we can assume that $0<\alpha_{n}<1 / 9<\alpha_{n-1} \leq \alpha_{n-2} \leq \cdots \leq \alpha_{1}<2 / 9$ and $\alpha_{1}+\alpha_{n}>2 / 9$.

Let $U_{1}$ be a sub-multiset of $S$ with minimal $\sum\left(U_{1}\right) \in[4 / 9,5 / 9]$ and $T_{1}$ a submultiset of $S$ with maximal $\sum\left(T_{1}\right)<4 / 9$.

If $\alpha_{n} \in U_{1}$, the claim follows immediately by taking $S_{1}^{\prime \prime}=U_{1}$ and $S_{1}^{\prime}=U_{1} \backslash\left\{\alpha_{n}\right\}$. If there is $\alpha_{i}<\alpha_{j}, i \neq n$, such that $\alpha_{i} \notin U_{1}$ and $\alpha_{j} \in U_{1}$, the claim follows by taking $S_{1}^{\prime \prime}=U_{1}$ and $S_{1}^{\prime}=U_{1} \cup\left\{\alpha_{i}\right\} \backslash\left\{\alpha_{j}\right\}$. Thus we can assume that $U_{1}=\left\{\alpha_{k}, \alpha_{k+1}, \ldots, \alpha_{n-1}\right\}$ for some $k$. Similarly we can assume that $T_{1}=\left\{\alpha_{1}, \alpha_{2}, \ldots, \alpha_{l}, \alpha_{n}\right\}$ for some $l$. By our assumptions on the sizes of $\alpha_{i}$ we have $\alpha_{n}+\alpha_{1}+\alpha_{2}+\alpha_{3}>2 / 9+1 / 9+1 / 9=4 / 9$, so $T_{1}=\left\{\alpha_{1}, \alpha_{n}\right\}$ or $T_{1}=\left\{\alpha_{1}, \alpha_{2}, \alpha_{n}\right\}$. But the first case cannot hold, since otherwise the fact that $\alpha_{1}+\alpha_{n}<\alpha_{1}+\alpha_{2}<4 / 9$ contradicts the maximality of $T_{1}$. Thus

$$
T_{1}=\left\{\alpha_{1}, \alpha_{2}, \alpha_{n}\right\}
$$

Since

$$
0<1-\sum\left(U_{1}\right)-\sum\left(T_{1}\right)<1-4 / 9-(2 / 9+1 / 9)=2 / 9,
$$

we see that

$$
S=U_{1} \dot{\cup}\left\{\alpha_{3}\right\} \dot{\cup} T_{1}
$$

Here

$$
\alpha_{3}=1-\sum\left(U_{1}\right)-\sum\left(T_{1}\right)<1-4 / 9-2 / 9-\alpha_{3}
$$

which implies that $\alpha_{3}<1 / 6$.

Let $S^{\prime}=\left\{\alpha_{2}, \alpha_{4}, \alpha_{5}, \alpha_{n}\right\}$. Then

$$
\begin{aligned}
\sum\left(S^{\prime}\right) & =\sum\left(T_{1}\right)-\alpha_{1}+\alpha_{4}+\alpha_{5} \\
& <\sum\left(T_{1}\right)+\alpha_{n}-2 / 9+2 \alpha_{3}<\sum\left(T_{1}\right)+1 / 9+\alpha_{n}
\end{aligned}
$$

Thus

$$
\sum\left(S^{\prime}\right) \in\left(\sum\left(T_{1}\right), \sum\left(T_{1}\right)+1 / 9+\alpha_{n}\right) .
$$


If $\sum\left(S^{\prime}\right) \leq 4 / 9$, this contradicts the choice of $T_{1}$. Otherwise the proof is completed by choosing $S_{1}^{\prime}=T_{1}$ and

$$
S_{1}^{\prime \prime}= \begin{cases}S^{\prime} & \text { if } \sum\left(S^{\prime}\right) \leq \sum\left(T_{1}\right)+1 / 9, \\ S^{\prime} \backslash\left\{\alpha_{n}\right\} & \text { else. }\end{cases}
$$

4. Averages of type II exponential sums. In this section we consider an average of the exponential sum

$$
S(x)=\sum_{n \sim X} a_{n} e(n x)=\sum_{\substack{m l \sim X \\ m \sim M}} b_{m} c_{l} e(m l x)
$$

over a certain set. Let $M, Q \in[1, X]$ and $\beta \leq Q^{-2}$. We use the following notation. The set $\mathcal{Q} \subset[Q, 2 Q)$ consists of distinct integers. For each $q \in \mathcal{Q}, a(q)$ is an integer satisfying $(a(q), q)=1$ and $\beta_{q}$ is a real number satisfying $\left|\beta_{q}\right| \sim \beta$. Then we define

$$
\alpha_{q}=\frac{a(q)}{q}+\beta_{q}
$$

and are interested in bounding

$$
S=\sum_{q \in \mathcal{Q}}\left|S\left(\alpha_{q}\right)\right|
$$

Further, we write $\beta^{\prime}=\max \{\beta, 1 / X\}$. By partial summation we can indeed assume in the proofs of the following lemmata that $\left|\beta_{q}\right| \sim \beta^{\prime}$. The following result improves [7, Lemma 3] for type II sums.

LEMMA 12. We have

$$
S^{2} \ll|\mathcal{Q}|^{2} X^{2+\eta} \beta^{\prime}\left(M+\frac{Q^{2}}{M}\right)+|\mathcal{Q}| X^{3+\eta} \beta^{\prime}\left(\frac{1}{Q}+\frac{1}{M}\right) .
$$

Proof. The method of the proof is similar to that of [7, Lemma 3]. Actually, we follow that proof until (30) of [7] to get

$$
S \ll \max _{|t| \leq X^{10}} X^{1 / 2+\eta} \beta^{\prime 1 / 2} \sum_{h \leq 2 Q} \sum_{q \in \mathcal{Q}} \sum_{\substack{m \sim M \\(q, m)=h}}\left|\sum_{l \leq X / M} c_{l} l^{-i t} e\left(\frac{m l a(q)}{q}\right)\right|+|\mathcal{Q}| X^{\eta} .
$$

Instead of using the Cauchy-Schwarz inequality here directly as in [7, Lemma 3], we first divide the summation over pairs $(q, m) \in \mathcal{Q} \times[M, 2 M]$ into sets $\mathcal{A}_{\delta_{i+1}, h} \backslash \mathcal{A}_{\delta_{i}, h}$ for $\delta_{i}=2^{i} / Q, \delta_{i} \in\left[h^{2} /\left(4 Q^{2}\right), 1\right]$, where $\mathcal{A}_{\delta_{j}, h}$ is defined as in Lemma 9. Then we have 
by the large sieve

$$
\begin{aligned}
& \sum_{(q, m) \in \mathcal{A}_{\delta_{i+1}, h} \backslash \mathcal{A}_{\delta_{i}, h}}\left|\sum_{l \leq X / M} c_{l} l^{-i t} e\left(\frac{m l a(q)}{q}\right)\right|^{2} \\
& \ll\left(\frac{M}{Q}+1\right)\left(\frac{1}{\delta_{i}}+\frac{X}{M}\right) \frac{X^{1+\eta}}{M} \\
\ll & \left(\frac{1}{Q}+\frac{1}{M}\right)\left(\frac{1}{\delta_{i}}+\frac{X}{M}\right) X^{1+\eta}
\end{aligned}
$$

By Lemma 9 and an elementary argument we have

$$
\sum_{h \leq 2 Q}\left|\mathcal{A}_{\delta_{i}, h}\right|^{1 / 2} \ll X^{\eta} \min \left\{|\mathcal{Q}|\left((M+Q) Q \delta_{i}\right)^{1 / 2},(|\mathcal{Q}| M)^{1 / 2}\right\} .
$$

Hence by the Cauchy-Schwarz inequality we get

$$
\begin{aligned}
S^{2} & \ll X^{2+\eta} \beta^{\prime}\left(\frac{1}{Q}+\frac{1}{M}\right) \cdot \max _{\frac{1}{4 Q^{2}} \leq \delta^{\prime} \leq 1}\left\{\left(\frac{1}{\delta^{\prime}}+\frac{X}{M}\right) \cdot \min \left\{|\mathcal{Q}|^{2}(M+Q) Q \delta^{\prime},|\mathcal{Q}| M\right\}\right\} \\
& \ll X^{2+\eta} \beta^{\prime}\left(\frac{1}{Q}+\frac{1}{M}\right)\left(|\mathcal{Q}|^{2}(M+Q) Q+|\mathcal{Q}| X\right),
\end{aligned}
$$

which implies the claim.

The previous lemma is useful when $Q$ is large. For smaller values of $Q$, we will use the following lemma.

LEMMA 13. We have

$$
S^{2} \ll|\mathcal{Q}|^{2} Q X^{2+\eta} \beta^{\prime 2}+\frac{|\mathcal{Q}| X^{1+\eta}}{Q} \max _{1 \leq T^{\prime} \leq X \beta^{\prime}} \frac{1}{T^{\prime}}\left(M+Q T^{\prime}\right)\left(\frac{X}{M}+Q^{2} T^{\prime}\right) .
$$

Proof. We use the identity

$$
\frac{1}{\phi(q)} \sum_{\chi(\bmod q)} \tau(\chi) \bar{\chi}(m)= \begin{cases}e(m / q) & \text { if }(m, q)=1 \\ 0 & \text { else }\end{cases}
$$

where

$$
\tau(\chi)=\sum_{k(\bmod q)} e\left(\frac{k}{q}\right) \chi(k)
$$

is the Gauss sum. This gives

$$
\left|S\left(\alpha_{q}\right)\right| \leq \sum_{g \mid q} \sum_{\chi(\bmod q / g)} \frac{|\tau(\chi)|}{\phi(q / g)}\left|\sum_{n \sim X / g} \chi(n) a_{n g} e\left(n g \beta_{q}\right)\right| .
$$


Changing to primitive characters leads to

$$
\begin{aligned}
\left|S\left(\alpha_{q}\right)\right| & \leq \sum_{r g \mid q} \frac{r^{1 / 2}}{\phi(q / g)} \sum_{\chi(\bmod r)}^{*}\left|\sum_{\substack{n \sim X / g \\
\left(n, \frac{q}{r g}\right)=1}} \chi(n) a_{n g} e\left(n g \beta_{q}\right)\right| \\
& \leq \sum_{r g d \mid q} \frac{r^{1 / 2}}{\phi(q / g)} \sum_{\chi(\bmod r)}^{*}\left|\sum_{n \sim X /(g d)} \chi(n) a_{n g d} e\left(n g d \beta_{q}\right)\right| \\
& \ll X^{\eta} \sum_{r g \mid q} \frac{g r^{1 / 2}}{q} \sum_{\chi(\bmod r)}^{*}\left|\sum_{n \sim X / g} \chi(n) a_{n g} e\left(n g \beta_{q}\right)\right| .
\end{aligned}
$$

We write $c=1+(\log X)^{-1}$ and $T=X^{2}$. Then by the Perron formula and partial integration

$$
\begin{aligned}
\sum_{n \sim X / g} \chi(n) a_{n g} e\left(n g \beta_{q}\right)= & \int_{X / g}^{2 X / g} e\left(\beta_{q} g y\right) \frac{1}{2 \pi i} \int_{c-i T}^{c+i T} \sum_{n \sim X / g} \frac{\chi(n) a_{n g}}{n^{s}} y^{s-1} d s d y \\
& +O\left(X^{1+\eta} \beta^{\prime}\right) .
\end{aligned}
$$

The error term contributes to $S$ the first term in (14). Thus we can assume that the main term dominates here.

We write

$$
h(s)=\int_{X / g}^{2 X / g} e\left(\beta_{q} g y\right) y^{s-1} d y .
$$

Then $h(s)$ is an entire function of $s$ and for $s=\sigma+i t$ we have by the first and second derivative tests ([12, Lemma 8.10] and partial summation)

$$
h(s) \ll \begin{cases}\left(\frac{X}{g}\right)^{\sigma} \min \left\{1,|t|^{-1 / 2}\right\} & \text { if }|t|<8 \pi X \beta^{\prime}, \\ \left(\frac{X}{g}\right)^{\sigma} \min \left\{1,|t|^{-1}\right\} & \text { if }|t| \geq 8 \pi X \beta^{\prime} .\end{cases}
$$

Hence

$$
\begin{aligned}
& S \ll \max _{1 \leq T^{\prime} \leq X^{2}} \frac{X^{1+\eta}}{T^{\prime 1 / 2}} \sum_{g r \leq 2 Q} \sum_{\substack{q \in \mathcal{Q} \\
g r \mid q}} \frac{r^{1 / 2}}{q} \sum_{\chi(\bmod r)}^{*} \int_{c+i T^{\prime}}^{c+2 i T^{\prime}}\left|\sum_{n \sim X / g} \frac{\chi(n) a_{n g}}{n^{s}}\right||d s|
\end{aligned}
$$

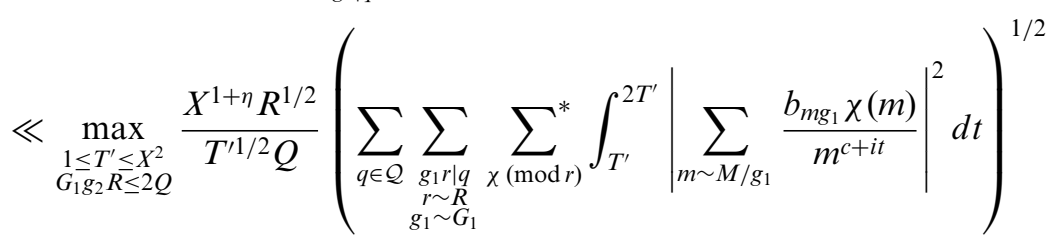

$$
\begin{aligned}
& \times\left(\frac{Q}{R} \sum_{r \sim R} \sum_{\chi(\bmod r)}^{*} \int_{T^{\prime}}^{2 T^{\prime}}\left|\sum_{l \sim \frac{X}{M g_{2}}} \frac{c_{g_{2}} \chi(l)}{l^{c+i t}}\right|^{2} d t\right)^{1 / 2}
\end{aligned}
$$


by the Cauchy-Schwarz inequality. Applying (10) and (11), we get

$$
\begin{aligned}
S & \ll \max _{\substack{1 \leq T^{\prime} \leq X^{2} \\
g_{1} g_{2} R \leq 4 Q}} \frac{X^{1 / 2+\eta}\left(g_{1} g_{2}\right)^{1 / 2} H^{1 / 2}}{T^{\prime 1 / 2} Q^{1 / 2}}\left(\frac{M}{g_{1}}+R T^{\prime}\right)^{1 / 2}\left(\frac{X}{M g_{2}}+R^{2} T^{\prime}\right)^{1 / 2} \\
& \ll \max _{1 \leq T^{\prime} \leq X^{2}} \frac{X^{1 / 2+\eta} H^{1 / 2}}{T^{\prime 1 / 2} Q^{1 / 2}}\left(M+Q T^{\prime}\right)^{1 / 2}\left(\frac{X}{M}+Q^{2} T^{\prime}\right)^{1 / 2} .
\end{aligned}
$$

The bound (15) allows us to replace $T^{\prime 1 / 2}$ in the denominator by $T^{\prime}$ for $T^{\prime} \geq 4 X \beta^{\prime}$ and thus we can assume that the maximum is attained for some $T^{\prime} \leq 4 X \beta^{\prime}$.

5. Large values of type II exponential sums. In this section, we apply the general results in Section 4 to our specific needs. Recall that $\epsilon=X^{-\xi+\delta}=X^{-2 / 9+\delta}$. The definitions in the beginning of the previous section still hold. Besides, we now assume that $\left|S\left(\alpha_{q}\right)\right| \geq Z$ for each $q \in \mathcal{Q}$. We also assume that

$$
Z \geq \epsilon X^{1-\eta}, \quad Q \leq\left(\frac{X^{1+\eta}}{Z}\right)^{2} \quad \text { and } \beta^{\prime} \ll \frac{X^{1+\eta}}{Q Z^{2}} .
$$

Our aim is to bound $|\mathcal{Q}|$. The lemmata in the previous section give bounds for $|\mathcal{Q}| Z$ and imply bounds for $|\mathcal{Q}|$ in many cases.

Lemma 14. Assume that $M \in\left[Q, Q X^{1 / 9}\right]$. Then

$$
|\mathcal{Q}| \ll \frac{X^{4+\eta}}{Z^{4} Q^{2}}
$$

Proof. By Lemma 12 we have

$$
\begin{aligned}
(|\mathcal{Q}| Z)^{2} & \ll|\mathcal{Q}|^{2} X^{2+\eta} \beta^{\prime}\left(M+\frac{Q^{2}}{M}\right)+|\mathcal{Q}| X^{3+\eta} \beta^{\prime}\left(\frac{1}{Q}+\frac{1}{M}\right) \\
& \ll|\mathcal{Q}|^{2} X^{19 / 9+\eta} \beta^{\prime} Q+\frac{|\mathcal{Q}| X^{3+\eta} \beta^{\prime}}{Q} .
\end{aligned}
$$

By our assumptions on $Z$ and $\beta^{\prime}$, the first term cannot dominate. Thus

$$
|\mathcal{Q}| \ll \frac{X^{3+\eta} \beta^{\prime}}{Z^{2} Q} \ll \frac{X^{4+\eta}}{Z^{4} Q^{2}} .
$$

Lemma 15. Assume that $M \in\left[X^{1 / 3}, X^{4 / 9}\right]$. Then

$$
|\mathcal{Q}| \ll \frac{X^{2+\eta}}{Q Z^{2}}+\frac{X^{13 / 9+\eta} Q}{Z^{2}} .
$$


Proof. By Lemma 13 we have

$$
\begin{aligned}
(|\mathcal{Q}| Z)^{2} & \ll \frac{|\mathcal{Q}| X^{1+\eta}}{Q} \max _{1 \leq T^{\prime} \leq X \beta^{\prime}} \frac{1}{T^{\prime}}\left(M+Q T^{\prime}\right)\left(\frac{X}{M}+Q^{2} T^{\prime}\right) \\
& \ll|\mathcal{Q}| X^{1+\eta}\left(\frac{X}{Q}+X^{4 / 9} Q+X^{2 / 3}+Q^{2} \beta^{\prime} X\right) \ll|\mathcal{Q}| X^{1+\eta}\left(\frac{X}{Q}+X^{4 / 9} Q\right),
\end{aligned}
$$

which implies the claim.

6. Proof of Proposition 6. The last assertion of Proposition 6 follows as [2, Lemma 7]. To prove the first assertion, we adapt the method of Section 3 of [7]. We use our improved results for averaged exponential sums and we also need to develop a different argument for the case, where both $\left|S_{i}(x)\right|$ are large.

We consider first the more difficult case that both $S_{1}(x)$ and $S_{2}(x)$ are type II sums. We define $\mathcal{A}_{1}$ to be the subset of the interval [1,P] satisfying the condition (i) of Proposition 6. Let $\mathcal{A}=[1, P] \backslash \mathcal{A}_{1}$ and $\mathcal{A}\left(Z_{1}, Z_{2}, Z_{3}\right)$ be the subset of $\mathcal{A}$ satisfying $\left|S_{j}(x)\right| \sim Z_{j}$ for $j=1,2,3$. Clearly, we can assume that

$$
Z_{1} \geq Z_{2} \geq \epsilon X^{1-\eta}
$$

Then by (7) and Dirichlet's theorem in Diophantine approximation, for each $x \in$ $\mathcal{A}\left(Z_{1}, Z_{2}, Z_{3}\right)$, there exist integers $a_{1}, q_{1}, a_{2}, q_{2}$ depending on $x$ such that

$$
\left|q_{j} \lambda_{j} x-a_{j}\right| \ll \frac{X^{1+\eta}}{Z_{j}^{2}}, \quad\left(a_{j}, q_{j}\right)=1, a_{j} \neq 0
$$

and

$$
q_{j} \ll \frac{X^{2+\eta}}{Z_{j}^{2}} .
$$

We let $\mathcal{A}^{\prime}=\mathcal{A}\left(Z_{1}, Z_{2}, Z_{3}, Q_{1}, Q_{2}, k\right)$ be the subset of $\mathcal{A}\left(Z_{1}, Z_{2}, Z_{3}\right)$ for which $q_{j}(x) \sim Q_{j}$ and $a_{j} \asymp k Q_{j}$. To prove the proposition we need to show that for every combination of $Z_{1}, Z_{2}, Z_{3}, Q_{1}, Q_{2}$ and $k$ that can arise either

$$
Z_{1}^{2} Z_{2}^{2} \mu\left(\mathcal{A}^{\prime}\right) \min \left\{\epsilon^{2}, k^{-2}\right\} \ll X^{3-\eta} \epsilon^{3}
$$

or

$$
Z_{1} Z_{2} Z_{3} \mu\left(\mathcal{A}^{\prime}\right) \min \left\{\epsilon^{2}, k^{-2}\right\} \ll X^{2-\eta} \epsilon^{2} .
$$

Thus we are led to estimate the Lebesgue measure $\mu\left(\mathcal{A}^{\prime}\right)$ of $\mathcal{A}^{\prime}$. First, we notice that for each $x \in \mathcal{A}^{\prime}$ we have

$$
\begin{aligned}
\left|a_{2} q_{1} \frac{\lambda_{1}}{\lambda_{2}}-a_{1} q_{2}\right| & =\left|q_{1} q_{2}\left(\frac{a_{2} / q_{2}}{\lambda_{2} x}\left(\lambda_{1} x-\frac{a_{1}}{q_{1}}\right)-\frac{a_{1} / q_{1}}{\lambda_{2} x}\left(\lambda_{2} x-\frac{a_{2}}{q_{2}}\right)\right)\right| \\
& \ll X^{1+\eta} \max \left\{\frac{Q_{1}}{Z_{2}^{2}}, \frac{Q_{2}}{Z_{1}^{2}}\right\}=\theta,
\end{aligned}
$$

say. 
Let $\mathcal{Q}_{1}$ be the set of $q_{1}$ such that $\left|S_{1}(x)\right|$ can be as large as $Z_{1}$. By Lemma 10 the inequality

$$
\left\|a_{2} q_{1} \frac{\lambda_{1}}{\lambda_{2}}\right\| \leq \theta, \quad q_{1} \in \mathcal{Q}_{1}, a_{2} \asymp k Q_{2}
$$

has

$$
H \ll\left|\mathcal{Q}_{1}\right| k Q_{2} \theta+q^{\prime \eta}\left(Q_{1}+k Q_{1} Q_{2} q^{\prime-1}+q^{\prime} \theta\right)
$$

solutions. Then $\mathcal{A}^{\prime}$ consists of $\ll H X^{\eta}$ intervals of at most length

$$
\min \left\{\frac{X^{1+\eta}}{Z_{1}^{2} Q_{1}}, \frac{X^{1+\eta}}{Z_{2}^{2} Q_{2}}\right\}=\gamma,
$$

say. We notice that

$$
\theta \gamma=\frac{X^{2+\eta}}{Z_{1}^{2} Z_{2}^{2}}
$$

We split into cases according to which term dominates in (20).

Case 1: $H \ll q^{1+\eta} \theta$. In this case

$$
Z_{1}^{2} Z_{2}^{2} \mu\left(\mathcal{A}^{\prime}\right) \min \left\{\epsilon^{2}, k^{-2}\right\} \ll X^{2+\eta} \epsilon^{2} q^{\prime} \ll X^{2+3 \delta+\eta} \epsilon^{-1} \ll X^{3-\eta} \epsilon^{3},
$$

which proves (18).

Case 2: $H \ll q^{\prime \eta}\left(Q_{1}+k Q_{1} Q_{2} q^{-1}\right)$. If $k>\epsilon^{-1} X^{\eta}$, then the left-hand side of (19) is

$$
\ll X^{1-\eta} \epsilon^{2} \frac{Z_{2} Z_{3}}{Z_{1}}+\epsilon X^{1-\eta} \frac{Q_{2} Z_{2} Z_{3}}{Z_{1} q^{\prime}} \ll X^{2-\eta} \epsilon^{2} .
$$

So we can assume that $k \leq \epsilon^{-1} X^{\eta}$ in which case $k Q_{1} Q_{2} q^{\prime-1} \ll Q_{1}$. Thus $H \ll$ $q^{\prime \prime} Q_{1}$. Then the left-hand side of (18) is

$$
\ll X^{1+\eta} \epsilon^{2} \min \left\{Z_{2}^{2}, Z_{1}^{2} \frac{Q_{1}}{Q_{2}}\right\},
$$

which is $\ll X^{3-\eta} \epsilon^{3}$ if $Z_{2}<X^{1-\eta} \epsilon^{1 / 2}$. Thus we can assume that

$$
Z_{1} \geq Z_{2} \geq X^{1-\eta} \epsilon^{1 / 2}
$$

which implies that

$$
Q_{j} \ll \frac{X^{2+\eta}}{Z_{j}^{2}} \ll \epsilon^{-1} X^{\eta} .
$$

On the other hand, the left-hand side of (19) is

$$
\ll X^{1+\eta} \epsilon^{2} \frac{Z_{2} Z_{3}}{Z_{1}}
$$

which is $\ll X^{2-\eta} \epsilon^{2}$ if $Z_{3} \ll X^{1-\eta}$. So we can further assume that $Z_{3} \geq X^{1-\eta}$. Then we find by (8) and Dirichlet's theorem in Diophantine approximation integers $q_{3} \leq X^{\eta}$ 
and $a_{3} \neq 0$ with $\left(a_{3}, q_{3}\right)=1$ such that

$$
\left|q_{3} \lambda_{3} x-a_{3}\right| \ll \frac{X^{1+\eta}}{Z_{3}^{2}} .
$$

As above we have

$$
\left|a_{3} q_{1} \frac{\lambda_{1}}{\lambda_{3}}-a_{1} q_{3}\right| \ll X^{1+\eta} \max \left\{\frac{q_{3}}{Z_{1}^{2}}, \frac{Q_{1}}{Z_{3}^{2}}\right\} \ll \epsilon^{-1} X^{\eta-1},
$$

where the last estimate follows from (22), (23) and bounds for $Z_{3}$ and $q_{3}$. Since

$$
a_{3} q_{1} \ll k q_{3} Q_{1} \leq \epsilon^{-2} X^{\eta}=o\left(X^{1-\eta} \epsilon\right),
$$

the number $\frac{a_{1} q_{3}}{a_{3} q_{1}}$ is a convergent to $\lambda_{1} / \lambda_{3}$. This implies that there are $\ll X^{\eta}$ possibilities for $\frac{a_{1} q_{3}}{a_{3} q_{1}}$. Say it is $a_{0} / q_{0}$, where $\left(a_{0}, q_{0}\right)=1$. Then

$$
\left\{\begin{array}{l}
a_{1} q_{3}=l a_{0} \\
a_{3} q_{1}=l q_{0}
\end{array}\right.
$$

Here $\left(q_{1}, q_{0} q_{3}\right)=\left(q_{1}, q_{0} a_{1} q_{3}\right)=\left(q_{1}, q_{0} l a_{0}\right)=q_{1}$ and thus $q_{1} \mid q_{0} q_{3}$. Hence there are $\ll X^{\eta}$ possibilities for $q_{1}$ and similarly for $q_{2}$.

Consider then the inequality

$$
\left|a_{2} q_{1} \frac{\lambda_{1}}{\lambda_{2}}-a_{1} q_{2}\right| \ll \theta .
$$

Consider solutions with $\operatorname{gcd}\left(a_{1}, a_{2}\right)=a_{g} \sim A$. If we had $\theta / a_{g}=o\left(1 / q^{\prime}\right)$ and $k Q_{1} Q_{2} / a_{g}=o\left(q^{\prime}\right)$, this would give too good a convergent to $\lambda_{1} / \lambda_{2}$ by the law of the best approximation. Thus we can assume that

$$
A \ll X^{\eta} \theta q^{\prime}+\frac{X^{\eta} k Q_{1} Q_{2}}{q^{\prime}} .
$$

By (22) and (23) we have

$$
\theta=X^{1+\eta} \max \left\{\frac{Q_{1}}{Z_{2}^{2}}, \frac{Q_{2}}{Z_{1}^{2}}\right\} \ll \frac{X^{3+\eta}}{\left(Z_{2} Z_{2}\right)^{2}} \ll X^{\eta-1} \epsilon^{-2}=o\left(X^{\eta} \epsilon^{2}\right)=o\left(1 /\left(k Q_{2}\right)\right),
$$

so (24) implies

$$
\left|q_{1} \frac{\lambda_{1}}{\lambda_{2}}-\frac{a_{1} q_{2}}{a_{2}}\right|<\frac{1}{2}\left(\frac{a_{g}}{k Q_{2}}\right)^{2}
$$

Thus $a_{1} q_{2} / a_{2}$ is a convergent to $q_{1} \lambda_{1} / \lambda_{2}$, and so there are $\ll A X^{\eta}$ possible triples $\left(a_{1}, q_{2}, a_{2}\right)$. This implies that $H \ll A X^{\eta}$. If the first term dominates in the estimate (25), we are led back in Case 1. Thus we can assume that the second term dominates in (25). Then the left-hand side of (18) is

$$
\ll \gamma X^{\eta} \frac{Q_{1} Q_{2}}{q^{\prime}} \epsilon\left(Z_{1} Z_{2}\right)^{2} \ll \frac{X^{1+\eta} \epsilon}{q^{\prime}} \min \left\{Q_{2} Z_{2}^{2}, Q_{1} Z_{1}^{2}\right\} \ll \frac{X^{3+\eta} \epsilon}{q^{\prime}} \ll X^{3-\eta} \epsilon^{3},
$$

where the penultimate estimate follows from (17). 
Case 3: $H \ll\left|\mathcal{Q}_{1}\right| k Q_{2} \theta$. In this case, we use the lemmata in Section 5 in order to estimate $\left|\mathcal{Q}_{1}\right|$.

Case $3 a: Z_{1}>X^{1-\eta} \epsilon^{1 / 2}$. Now $Q_{1} \leq X^{\eta} \epsilon^{-1}$. If $Q_{1} Q_{2} \leq X^{1-\eta} \epsilon^{2}$, we have by the trivial estimate $\left|\mathcal{Q}_{1}\right| \leq Q_{1}$ that the left-hand side of (18) is

$$
\ll \gamma H Z_{1}^{2} Z_{2}^{2} \min \left\{\epsilon^{2}, k^{-2}\right\} \ll X^{2+\eta} Q_{1} Q_{2} \epsilon \ll X^{3-\eta} \epsilon^{3} .
$$

Thus we can assume that $Q_{1} \gg X^{1-\eta} \epsilon^{2} / Q_{2}$.

By Lemma 15 we have

$$
\left|\mathcal{Q}_{1}\right| \ll \frac{X^{2+\eta}}{Q_{1} Z_{1}^{2}}+\frac{X^{13 / 9+\eta} Q_{1}}{Z_{1}^{2}} .
$$

Thus,

$$
\begin{aligned}
\gamma H Z_{1}^{2} Z_{2}^{2} \min \left\{\epsilon^{2}, k^{-2}\right\} & \ll \frac{X^{4+\eta} Q_{2} \epsilon}{Q_{1} Z_{1}^{2}}+\frac{X^{31 / 9+\eta} Q_{1} Q_{2} \epsilon}{Z_{1}^{2}} \\
& \ll \frac{X^{1+\eta} Q_{2}^{2}}{\epsilon^{2}}+X^{13 / 9+\eta} Q_{1} Q_{2} \\
& \ll \frac{X^{1+\eta}}{\epsilon^{6}}+\frac{X^{13 / 9+\eta}}{\epsilon^{3}} \ll X^{3-\eta} \epsilon^{3} .
\end{aligned}
$$

Case $3 b: \max \left\{Z_{1}, Z_{2}\right\} \leq X^{1-\eta} \epsilon^{1 / 2}$. We can argue as in the beginning of this section with roles of $q_{1}$ and $q_{2}$ swapped to conclude that we can assume that

$$
H \ll k \theta \min \left\{\left|\mathcal{Q}_{1}\right| Q_{2}, Q_{1}\left|\mathcal{Q}_{2}\right|\right\} .
$$

We renumber such that $Q_{1} \geq Q_{2}$ (we do not anymore assume that $Z_{1} \geq Z_{2}$ ).

For $Q_{2} \leq Q_{1} \leq X^{1 / 3}$ the expression on the first line of (26) is

$$
\ll \frac{X^{4+\eta} \epsilon}{Z_{1}^{2}}+\frac{X^{31 / 9+\eta} Q_{1}^{2} \epsilon}{Z_{1}^{2}} \ll \frac{X^{2+\eta}}{\epsilon}+\frac{X^{13 / 9+\eta} Q_{1}^{2}}{\epsilon} \ll X^{3-\eta} \epsilon^{3}
$$

by (16).

For $Q_{1} \geq X^{1 / 3}$ we use Lemma 14 giving

$$
\left|\mathcal{Q}_{1}\right| \ll \frac{X^{4+\eta}}{Z_{1}^{4} Q_{1}^{2}} .
$$

Thus the left-hand side of (18) is

$$
\ll X^{2+\eta} \epsilon Q_{2} \frac{X^{4}}{Z_{1}^{4} Q_{1}^{2}} \ll \frac{X^{2+\eta}}{\epsilon^{3} Q_{1}} \ll X^{3-\eta} \epsilon^{3}
$$

by (16) and we have proved Proposition 6 for type II sums.

If one or both of $S_{1}(x)$ and $S_{2}(x)$, say $S_{2}(x)$, (we do not assume at this point that $Z_{1} \geq Z_{2}$ or $Q_{1} \geq Q_{2}$ ) is a type I sum, we use (8) and Dirichlet's theorem in Diophantine approximation. They let us find integers $a_{2}, q_{2}$ depending on $x$ such that

$$
\left|q_{2} \lambda_{2} x-a_{2}\right| \ll \frac{X^{\eta}}{Z_{2}}, \quad\left(a_{2}, q_{2}\right)=1,
$$


and

$$
q_{2} \ll \frac{X^{1+\eta}}{Z_{2}}
$$

Then we have adopting the notation above that

$$
\theta=\max \left\{\frac{Q_{1}}{Z_{2}}, \frac{Q_{2} X}{Z_{1}^{2}}\right\} X^{\eta}, \quad \gamma=\min \left\{\frac{1}{Z_{2} Q_{2}}, \frac{X}{Z_{1}^{2} Q_{1}}\right\} X^{\eta}
$$

and

$$
\gamma \theta=\frac{X^{1+\eta}}{Z_{1}^{2} Z_{2}}
$$

By Lemma 10 the inequality

$$
\left\|a_{2} q_{1} \frac{\lambda_{1}}{\lambda_{2}}\right\| \leq \theta
$$

has at most

$$
\ll Q_{1} k Q_{2} \theta+q^{\prime \eta}\left(\min \left\{Q_{1}, Q_{2}\right\}+k Q_{1} Q_{2} q^{\prime-1}+q^{\prime} \theta\right)
$$

solutions. By the discussion in the beginning of this section, we can assume that the first term dominates here. Then we need to bound

$$
\begin{aligned}
& \gamma X^{\eta} Q_{1} k Q_{2} \theta Z_{1}^{2} Z_{2}^{2} \min \left\{\epsilon^{2}, k^{-2}\right\} \ll X^{\eta} Q_{1} Q_{2} Z_{1}^{2} Z_{2}^{2} \epsilon \frac{X}{Z_{1}^{2} Z_{2}} \\
& \ll X^{1+\eta} Q_{1}\left(Q_{2} Z_{2}\right) \epsilon \ll X^{2+\eta} Q_{1} \epsilon \ll X^{3-\eta} \epsilon^{3} .
\end{aligned}
$$

This concludes the proof of Proposition 6.

7. Sieve asymptotic formulae. The lower and upper bound functions $\rho^{+}(n)$ and $\rho^{-}(n)$ arise from applications of Buchstab's identity

$$
\rho(u, z)=\rho(u, w)-\sum_{w \leq p<z} \rho(u / p, p)
$$

where for $u \in \mathbb{N}, z>1$

$$
\rho(u, z)=\left\{\begin{array}{l}
1 \quad \text { if }(u, P(z))=1, \\
0 \quad \text { otherwise, }
\end{array} \quad \text { with } \quad P(z)=\prod_{p<z} p\right.
$$

and $\rho(u, z)=0$ for $u \notin \mathbb{N}$.

We have the following

Lemma 16. Let $K \leq X^{5 / 9}, L \leq X^{1 / 3}, K L \leq X^{7 / 9}$ and $z=X^{1 / 9}$. Assume further that $K(s)=\sum_{k \sim K} a_{k} k^{-s}$ and $L(s)=\sum_{l \sim L} b_{l} l^{-s}$ are products of $\ll 1$ Dirichlet polynomials of 
length $\leq X^{2 / 9}$. Then the sum

$$
\sum_{\substack{k l m \sim X \\ k \sim K, l \sim L}} a_{k} b_{l} \rho(m, z) e(k \operatorname{lm} x)
$$

can be written as a sum of $\ll(\log X)^{C}$ type I and type II sums.

Proof. We use Buchstab's identity with $w=1$ to decompose the sum into a sum of $\ll \log X$ sums of the form

$$
\sum_{\substack{k \operatorname{lm} p_{1} \cdots p_{j} \sim X \\ k \sim K, l \sim L \\ p_{j}<p_{j-1}<\cdots<p_{1}<z}} a_{k} b_{l} e\left(k \operatorname{lm} p_{1} \cdots p_{j} x\right) .
$$

We split each of these into $\ll \log X$ sums

$$
\sum_{\substack{k \operatorname{lmp} p_{1} \cdots p_{j} \sim X \\ k \sim K, l \sim L \\ z>p_{1}>p_{2}>\cdots>p_{j} \\ \cdots p_{i} \leq X^{4 / 9}<k p_{1} \cdots p_{i+1}}} a_{k} b_{l} e\left(k \operatorname{lmp} p_{1} \cdots p_{j} x\right) .
$$

In the case $i=j$, we have a type I sum since then $k l p_{1} \cdots p_{j} \leq X^{7 / 9}$. Otherwise, $K p_{1} \cdots p_{i+1} \in\left[X^{4 / 9}, X^{5 / 9}\right]$ and $K L p_{1} \cdots p_{i} \leq X^{7 / 9}$. We further split the sum to $\ll$ $(\log X)^{C}$ sums by combining $p_{i}$ to at most 20 variables in the interval $\left[X^{1 / 30}, X^{1 / 9}\right]$ (and at most one $\leq X^{1 / 30}$ ) and then restricting these in dyadic manner. Then we see from Lemma 11 that we have a type II sum. Possible cross-conditions in type II sums can be handled by the Perron formula as in [8, Lemma 1].

For $n \sim X$, we can write the characteristic function of primes as $\rho(n)=\rho\left(n, 2 X^{1 / 2}\right)$. Our aim is to give lower and upper bounds for this such that they are sums of type I and type II sums. To get a lower bound, we start by applying Buchstab's identity twice giving

$$
\begin{aligned}
\rho(n) & =\rho(n, z)-\sum_{z \leq p<2 \sqrt{X}} \rho\left(\frac{n}{p}, z\right)+\sum_{z \leq p_{2}<p_{1}<2 \sqrt{X}} \rho\left(\frac{n}{p_{1} p_{2}}, p_{2}\right) \\
& =a_{n, 1}-a_{n, 2}+a_{n, 3} .
\end{aligned}
$$

Using Heath-Brown's generalised Vaughan identity [10] we see that sums arising from applications of Buchstab's identity can always be split into sums of products, where all the terms of magnitude $\geq X^{2 / 9}$ have coefficients that arise from a characteristic function of an interval. Thus sums having terms of length $\geq X^{2 / 9}$ lead to type I sums. So by the previous lemma we can write the sum $\sum_{n \sim X} a_{n, i} e\left(\lambda_{j} n x\right)$ for $i=1,2$ as a sum of type I and type II sums.

We write $a_{n, 3}^{\prime}$ for the part of $a_{n, 3}$ with $p_{1} p_{2}^{2}<X$. We write $p_{i}=X^{\alpha_{i}}$. Some parts of $\sum_{n \sim X} a_{n, 3}^{\prime} e\left(\lambda_{j} n x\right)$ are already satisfactory type II sums. For example, in the part satisfying

$$
\alpha_{1}+\alpha_{2} \in[4 / 9,5 / 9] \text { and } \alpha_{1} \in[7 / 18,4 / 9] \text {, }
$$


we have $1-\alpha_{1}-\alpha_{2} \in[4 / 9,5 / 9], \alpha_{1} \in[1 / 3,4 / 9]$ and

$$
\left(1-\alpha_{1}-\alpha_{2}\right)-\alpha_{1} \leq 1-2 \cdot 7 / 18-1 / 9=1 / 9 .
$$

Thus we have a type II sum. We do not need to consider such areas further.

In certain areas we can use Buchstab's identity to decompose twice more

$$
\begin{aligned}
& \sum_{\substack{z \leq p_{2}<p_{1}<2 \sqrt{X} \\
\left(p_{1}, p_{2}\right) \in A}} \rho\left(\frac{n}{p_{1} p_{2}}, p_{2}\right)=\sum_{\substack{z \leq p_{2}<p_{1}<2 \sqrt{X} \\
\left(p_{1}, p_{2}\right) \in A}} \rho\left(\frac{n}{p_{1} p_{2}}, z\right) \\
& -\sum_{\substack{z \leq p_{3}<p_{2}<p_{1}<2 \sqrt{X} \\
\left(p_{1}, p_{2}\right) \in A, p_{1} p_{2} p_{3}^{2}<2 X}} \rho\left(\frac{n}{p_{1} p_{2} p_{3}}, z\right)+\sum_{\substack{z \leq p_{4}<p_{3}<p_{2}<p_{1}<2 \sqrt{X} \\
\left(p_{1}, p_{2}\right) \in A, p_{1} p_{2} p_{3}^{2}<2 X}} \rho\left(\frac{n}{p_{1} p_{2} p_{3} p_{4}}, p_{4}\right) .
\end{aligned}
$$

Since the second term on the right-hand side has a negative sign, we cannot discard it when we are looking for a lower bound for $\rho(n)$. So we can decompose this way only if that term can be written as a sum of type I and type II sums. This is the case, if we can combine $\alpha_{1}, \alpha_{2}$ and $\alpha_{3}^{(M)}=\min \left\{\alpha_{2}, \frac{1-\alpha_{1}-\alpha_{2}}{2}\right\}$ to two variables satisfying the conditions of Lemma 16. We can do this, for example, in the part, where $\alpha_{1}+2 \alpha_{2} \leq 7 / 9$ and $\alpha_{1}+\alpha_{2} \leq 5 / 9$. In some parts we can still decompose further.

Even if the conditions of Lemma 16 are not immediately satisfied, we can still decompose twice more in some parts. This is the case if for some $\alpha_{3}^{\prime}<\alpha_{3}^{(M)}$ we can combine $\alpha_{1}, \alpha_{2}$ and $\alpha_{3}^{\prime}$ to satisfy the conditions of Lemma 16 and for every $\alpha_{3} \in$ $\left(\alpha_{3}^{\prime}, \alpha_{3}^{(M)}\right)$ we are in type II area. This holds for example when $\alpha_{1} \leq 1 / 3$ and $\alpha_{1}+\alpha_{2} \in$ $[5 / 9,11 / 18]$ : Then, $\alpha_{3}^{(M)} \leq 2 / 9$ and we can take $\alpha_{3}^{\prime}=4 / 9-\alpha_{1}$.

Discarding the regions with a positive sign that are not type II sums and where we cannot decompose further, we are led to a lower bound $\rho^{-}(n) \leq \rho(n)$. We still need to show that (5) holds for an appropriate constant $u^{-}$. To that end, we write $\mathcal{A}=[y, y+y \vartheta)$ and $\mathcal{B}=\left[y, y+y \vartheta^{\prime}\right)$, where $\vartheta^{\prime}=\exp \left(-(\log X)^{4 / 7}\right)$. We will first show that

$$
\int_{d X}^{D X}\left(\sum_{m \in \mathcal{A}} \rho^{-}(m)-\frac{\vartheta}{\vartheta^{\prime}} \sum_{m \in \mathcal{B}} \rho^{-}(m)\right)^{2} d y \ll \frac{X}{\tau^{2}}(\log X)^{-A} .
$$

Clearly, it is enough to show that this holds when $\rho^{-}$is replaced by our type I and type II sums. If we have a type I sum with $M \leq X^{7 / 9}<X^{1-\eta} \vartheta$, this holds by an elementary argument. Thus we need to consider only type II sums $\sum_{m n \in \mathcal{A}, m \sim M} a_{m} b_{n}$ with $M \in\left[X^{4 / 9}, X^{5 / 9}\right]$. These kinds of integrals arise in considerations on primes in almost all short intervals (compare the following with Lemma 9.3 of [9]). Since the length of our interval is now long, $y \vartheta \asymp X^{7 / 9}$, the task is easy. We get by the Perron formula and changing the order of integrations that the left-hand side of (27) is for $T_{0}=\exp \left(\log ^{1 / 3} X\right)$ and $T=\vartheta^{-1} X^{\eta}$ apart from an admissible error

$$
\ll \tau^{-2} \int_{T_{0}}^{T}|F(1 / 2+i t)|^{2} d t \ll \tau^{-2} \max _{t \in\left[T_{0}, T\right]}\left|\sum_{m \sim M} a_{m} m^{-1 / 2-i t}\right|^{2}\left(\frac{X}{M}+T\right) \log ^{C} X .
$$


This is $\ll \frac{X}{\tau^{2}}(\log X)^{-A}$ assuming that

$$
\sum_{m \sim M} a_{m} m^{-1 / 2-i t} \ll M^{1 / 2}(\log X)^{-A}
$$

which holds in interesting cases since the coefficient arise from the characteristic function of primes. For results of this type, see for example [11, Lemma 19].

By (27) we obtain (5) if we can show that

$$
\begin{aligned}
\sum_{m \in \mathcal{B}} \rho^{-}(m) & =\frac{u^{-} \vartheta^{\prime}}{\vartheta} \sum_{m \in \mathcal{A}} \frac{1}{\log m}+O\left(X \exp \left(-2(\log X)^{1 / 3}\right)\right) \\
& =\frac{u^{-} y \vartheta^{\prime}}{\log y}+O\left(X \exp \left(-2(\log X)^{1 / 3}\right)\right)
\end{aligned}
$$

By the construction of the weights $\rho^{-}(m)$, we have

$$
\sum_{m \in \mathcal{B}} \rho^{-}(m)=\sum_{m \in \mathcal{B}} \rho(m)-\sum_{j} \rho_{j}^{\prime}(m),
$$

where the terms corresponding to discarded regions are of the form

$$
\rho_{j}^{\prime}(m)=\sum_{y^{\nu}<p_{j}<\cdots<p_{1}<y^{\lambda}} \rho\left(\frac{n}{p_{1} \cdots p_{j}}, p_{j}\right)
$$

with some additional summation conditions. By the prime number theorem

$$
\sum_{m \in \mathcal{B}} \rho(m)=\frac{\vartheta^{\prime} y}{\log y}(1+O(1)) .
$$

For other sums standard methods (see [9, Section 1.4]) give for example

$$
\begin{aligned}
& \sum_{n \in \mathcal{B}} \sum_{y^{\nu}<p_{j}<\cdots<p_{1}<y^{\lambda}} \rho\left(\frac{n}{p_{1} \cdots p_{j}}, p_{j}\right) \\
&= \frac{\vartheta^{\prime} y}{\log y} \int_{\alpha_{1}=v}^{\lambda} \int_{\alpha_{2}=v}^{\alpha_{1}} \cdots \int_{\alpha_{j}=v}^{\alpha_{j-1}} \omega\left(\frac{1-\alpha_{1}-\cdots-\alpha_{j}}{\alpha_{j}}\right) \frac{d \alpha_{j} \cdots d \alpha_{1}}{\alpha_{1} \cdots \alpha_{j-1} \alpha_{j}^{2}} \\
& \quad+O\left(y \exp \left(-2(\log y)^{1 / 3}\right)\right),
\end{aligned}
$$

where $\omega(u)$ is Buchstab's function.

Hence we find the constant $u^{-}$by subtracting from 1 the integrals corresponding to $\rho_{i}^{\prime}$. Employing a computer to do the decompositions and numerical integration, we are led to a lower bound consisting of type I and type II sums with $u^{-}>0.60$

Similarly, we find an upper bound by starting to apply Buchstab's identity three times. In the case of the upper bound, we can discard only terms with a negative sign. This leads to $u^{+}<1.19$. Thus $2 u^{-}-u^{+}>0$ and the proof of the theorem is completed.

ACKNOWLeDGEMENTS. This work was conducted while the author was a research student at Royal Holloway, University of London. She was supported by EPSRC 
grant GR/T20236/01. The author thanks Glyn Harman for his helpful comments and suggestions.

\section{REFERENCES}

1. A. Baker, On some Diophantine inequalities involving primes, J. Reine Angew. Math. 228 (1967), 166-181.

2. R. C. Baker and G. Harman, Diophantine approximation by prime numbers, J. Lond. Math. Soc. 25(2) (1982), 201-215.

3. R. C. Baker, G. Harman and J. Pintz, The exceptional set for Goldbach's problem in short intervals, in Sieve methods, exponential sums and their applications in number theory (Greaves, G. R. H., Harman, G. and Huxley, M. N., Editors) (Cambridge University Press, Cambridge, UK, 1997), 1-54.

4. J. Brüdern and E. Fouvry, Le crible à vecteurs, Compositio Math. 102 (1996), 337-355.

5. P. X. Gallagher, A large sieve density estimate near $\sigma=1$, Invent. Math. 11 (1970), $329-339$ $9-18$.

6. G. Harman, On the distribution of $\alpha p$ modulo one I, J. Lond. Math. Soc. 27(2) (1983),

7. G. Harman, Diophantine approximation by prime numbers, J. Lond. Math. Soc. 44(2) (1991), 218-226.

8. G. Harman, On the distribution of $\alpha p$ modulo one II, Proc. Lond. Math. Soc. 72(3) (1996), 241-260.

9. G. Harman, Prime-detecting sieves, vol. 33: London Mathematical Society Monographs (New Series) (Princeton University Press, Princeton, 2007).

10. D. R. Heath-Brown, Prime numbers in short intervals and a generalized Vaughan identity, Can. J. Math. 34 (1982), 1365-1377.

11. D. R. Heath-Brown, The number of primes in a short interval, J. Reine Angew. Math. 389 (1988), 22-63.

12. H. Iwaniec and E. Kowalski, Analytic number theory, vol. 53: American Mathematical Society Colloquium Publications (American Mathematical Society, Providence, RI, 2004).

13. B. Saffari and R. C. Vaughan, On the fractional parts of $x / n$ and related sequences II, Ann. Inst. Fourier 27 (1977), 1-30.

14. W. Schwarz, Über die Lösbarkeit gewisser Ungleichungen durch Primzahlen, J. Reine Angew. Math. 212 (1963), 150-157.

15. R. C. Vaughan, Diophantine approximation by prime numbers, I, Proc. Lond. Math. Soc. 28(3), (1974), 373-384.

16. R. C. Vaughan, The Hardy-Littlewood method, vol. 125: Cambridge Tracts in Mathematics, 2nd edition (Cambridge University Press, Cambridge, UK, 1997). 